\title{
Oxidative modification of caspase- 9 facilitates its activation via disulfide-mediated interaction with Apaf-1
}

\author{
Yong Zuo ${ }^{1, *}$, Binggang Xiang ${ }^{1, *}$, Jie Yang ${ }^{1}$, Xuxu Sun ${ }^{1}$, Yumei Wang ${ }^{1}$, Hui Cang ${ }^{1}$, Jing $\mathrm{Yi}^{1}$ \\ ${ }^{I}$ Department of Cell Biology, Key Laboratory of the Education Ministry for Cell Differentiation and Apoptosis, Institutes of Medi- \\ cal Sciences, Shanghai Jiao Tong University School of Medicine, Shanghai 200025, China
}

\begin{abstract}
Intracellular reactive oxygen species (ROS) are known to regulate apoptosis. Activation of caspase-9, the initial caspase in the mitochondrial apoptotic cascade, is closely associated with ROS, but it is unclear whether ROS regulate caspase-9 via direct oxidative modification. The present study aims to elucidate the molecular mechanisms by which ROS mediate caspase-9 activation. Our results show that the cellular oxidative state facilitates caspase-9 activation. Hydrogen peroxide treatment causes the activation of caspase-9 and apoptosis, and promotes an interaction between caspase-9 and apoptotic protease-activating factor 1 (Apaf-1) via disulfide formation. In addition, in an in vitro mitochondria-free system, the thiol-oxidant diamide promotes auto-cleavage of caspase-9 and the caspase-9/ Apaf-1 interaction by facilitating the formation of disulfide-linked complexes. Finally, a point mutation at $\mathbf{C} 403$ of caspase-9 impairs both $\mathrm{H}_{2} \mathrm{O}_{2}$-promoted caspase-9 activation and interaction with Apaf-1 through the abolition of

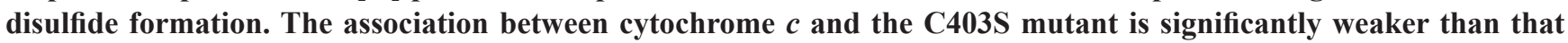
between cytochrome $c$ and wild-type caspase-9, indicating that oxidative modification of caspase-9 contributes to apoptosome formation under oxidative stress. Taken together, oxidative modification of caspase-9 by ROS can mediate its interaction with Apaf-1, and can thus promote its auto-cleavage and activation. This mechanism may facilitate apoptosome formation and caspase-9 activation under oxidative stress.
\end{abstract}

Keywords: oxidative modification, ROS, caspase-9, apoptosis, disulfide

Cell Research (2009) 19:449-457. doi: 10.1038/cr.2009.19; published online 24 February 2009

\section{Introduction}

Aerobic cells unavoidably produce reactive oxygen species (ROS) as a result of their metabolic activities and are consequently vulnerable to oxidative stress originating from the intra- or extracellular environments. Oxidative

\footnotetext{
*These two authors contributed equally to this work. Correspondence: Jing $\mathrm{Yi}^{\mathrm{a}}$, Jie Yang ${ }^{\mathrm{b}}$

${ }^{a}$ Tel: +86-21-63846590 ext. 776565

E-mail: yijing@shsmu.edu.cn

${ }^{\mathrm{b}}$ Tel: +86-21-63846590 ext. 776402

E-mail: yangiieyj@126.com

Abbreviations: $\beta$-ME (beta mercaptoethanol); $\mathrm{As}_{2} \mathrm{O}_{3}$ (arsenic trioxide); DTT (dithiothreitol); F5M (fluorescein-5-maleimide); FITC (fluorescein isothiocyanate); $\mathrm{H}_{2} \mathrm{O}_{2}$ (hydrogen peroxide); IP (immunoprecipitation); IB (immunoblot); Mn-SOD (manganese superoxide dismutase); MW (molecular weight); NAC ( $N$-acetyl-L-cysteine); OSC cells (oral squamous carcinoma cells); OSC-SA (OSC SOD-antisense); OSC-WT (OSC wild type); PI (propidium iodide); ROS (reactive oxygen species); TNF- $\alpha$ (tumor necrosis factor- $\alpha$ )

Received 4 August 2008; revised 13 October 2008; accepted 12 January 2009; published online 24 February 2009
}

stress is usually defined as a deteriorative process in which ROS modify numerous cellular components, including proteins, nucleic acids and lipids [1]. Although cells can counter and adapt to oxidative stress by activating diverse mechanisms that detoxify ROS and repair cellular damage, the major outcome of oxidative stress is cell death; modestly increased ROS levels induce apoptosis, whereas severe oxidative damage leads to necrosis [2]. Caspase-9 is an initial caspase in the mitochondrial apoptotic cascade that is activated in response to the insult of oxidative stress [3]. However, the precise mechanism by which caspase-9 molecules respond to increased ROS, specifically, the nature of the association between oxidative stress and caspase- 9 activation, remains controversial.

Caspases are named after their properties of acting as cysteine proteases that specifically cleave substrates after aspartic acid residues [4]. Because cysteines provide thiol groups to proteins and allow them to be reversibly modified by oxidants and reductants, the fundamental position of cysteine in the caspase family implies that these proteins may be redox-sensitive. The cysteines present in 
the catalytic sites of caspases are nucleophilic groups that usually must be kept in their reduced form to maintain enzyme activity. S-nitrosylation of these critical cysteine residues can inhibit caspase activity [5-7], and apoptotic stimuli, such as the Fas ligand, induce denitrosylation of the cysteine in the catalytic site of caspase-3, leading to its activation [6]. These data suggest that caspase activity requires catalytic cysteines to be in their reduced form [8].

On the other hand, growing evidence demonstrates that ROS serve as an important mediator in caspase-9dependent apoptotic processes [9-12]. Not only can intra- or extracellular oxidants directly induce apoptosis in which caspase-9 is inevitably activated, but also various drugs and cytokines can kill cells via the mitochondrial apoptotic pathway accompanied by ROS generation, and blockage of ROS rescues cells from caspase-9-dependent apoptosis [10-12]. Because many proteins undergo oxidative modifications during apoptotic events in which cysteines are oxidized to form disulfide bonds [9, 13], it has been suggested that caspase- 9 may be activated through oxidative modification. We found in our previous studies that the enzymatic activity of caspase- 9 was promoted by an intracellular ROS producer, emodin [14, 15]. These observations seem to conflict with the notion that the cysteines in the caspase catalytic site must be kept in a reduced form.

Caspase- 9 activation is initiated by the formation of apoptosomes. During apoptosis, cytochrome $c$ is released from mitochondria into the cytoplasm, in which it binds to apoptotic protease-activating factor 1 (Apaf-1) and, in the presence of dATP or ATP, these components assemble into a complex called the apoptosome [16, 17]. A recent study demonstrated caspase- 9 dimer formation in response to oxidative stress inside of the mitochondria, during which initiation of the intra-mitochondrial autocleavage of caspase- 9 preceded the onset of the caspase cascade in the cytosol [9]. This work has pointed out the essential role of oxidative modification in initiating caspase activation. In addition, it has been found that ROS promote apoptosome formation by oxidizing Apaf-1, consequently promoting activation of caspase- 9 and -3 [10]. These findings indicate that ROS may trigger caspase- 9 activation by pathways other than the stimulation of cytochrome $c$ release from mitochondria.

In the present study, we investigate the direct effects of ROS and the thiol-oxidizing agent diamide on caspase-9 molecules in an attempt to elucidate the molecular mechanisms by which oxidative stress induces caspase- 9 activation in the cytosol, regardless of actions derived from the mitochondria. The results demonstrate that $\mathrm{H}_{2} \mathrm{O}_{2}$ and diamide can oxidatively modify caspase- 9 , which facilitates the interaction of caspase- 9 and Apaf- 1 through the formation of disulfide bond within a complex and subsequent cleavage of caspase-9, ultimately promoting caspase- 9 activation. This finding explicates one of the fundamental mechanisms by which oxidative stress initiates cell apoptosis.

\section{Results}

\section{ROS facilitates caspase- 9 activation}

$\mathrm{As}_{2} \mathrm{O}_{3}$ has been reported to induce cell apoptosis through an ROS-dependent mechanism. As shown in Figure $1 \mathrm{~A}, \mathrm{As}_{2} \mathrm{O}_{3}$ treatment increased caspase- 9 activity. The increase could be prevented by $\mathrm{N}$-acetyl-L-cysteine (NAC) pre-treatment, indicating that ROS was required for $\mathrm{As}_{2} \mathrm{O}_{3}$-induced apoptosis. Interestingly, pre-treatment with NAC could also abolish caspase- 9 activity induced by TNF- $\alpha$, an extrinsic pro-apoptotic reagent. To further determine the role of ROS in apoptosis, we compared the caspase- 9 activity in TNF- $\alpha$-treated OSC-WT and OSCSA cells, two cell lines with different ROS levels. OSCSA cells exhibited decreased superoxide dismutase (SOD) expression and increased ROS levels relative to OSCWT cells (Figure 1B, upper panel). TNF- $\alpha$-induced caspase-9 activity was higher in the OSC-SA cells than in the OSC-WT cells. Similarly, NAC pre-treatment could abolish the activation of caspase- 9 induced by TNF- $\alpha$ in both of these cell lines (Figure 1B, lower panel). These
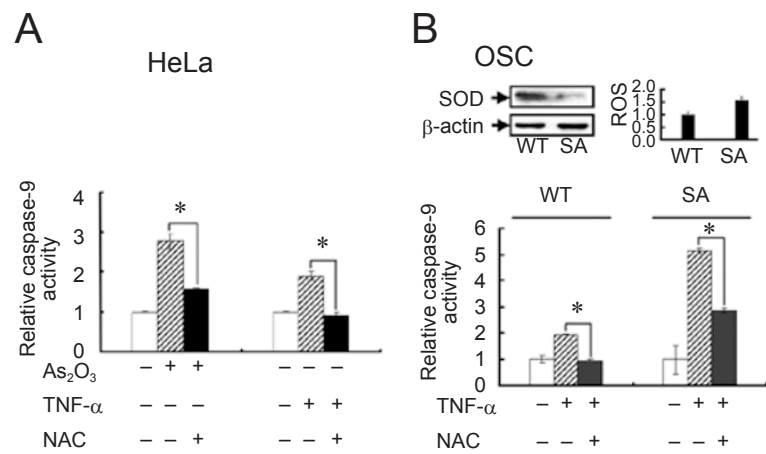

Figure 1 ROS facilitated caspase- 9 activation induced by arsenic trioxide and TNF- $\alpha$. (A) HeLa cells were stimulated by 0.5 $\mu \mathrm{M}$ arsenic trioxide $\left(\mathrm{As}_{2} \mathrm{O}_{3}\right)$ for $10 \mathrm{~h}$ or $10 \mathrm{ng} / \mathrm{ml} \mathrm{TNF}-\alpha$ for $20 \mathrm{~h}$. Cells were pre-incubated with $1.5 \mathrm{mM}$ NAC for $4 \mathrm{~h}$. Caspase- 9 activity was determined by the intensity of cleaved fluorescence-LEHD. (B) Oral squamous carcinoma (OSC) cells stably expressing antisense transcripts of manganese superoxide dismutase (Mn-SOD) were named OSC-SOD-antisense (OSCSA), whereas the parental OSC strain were named OSC-WT. Expression of SOD and ROS levels in OSC-SA cells and OSCWT cells were analyzed by immunoblot and DCFH flow cytometry (upper panel). OSC cells were treated with $10 \mathrm{ng} / \mathrm{ml}$ TNF- $\alpha$ for $20 \mathrm{~h}$ after pre-incubation with $3 \mathrm{mM}$ NAC for $4 \mathrm{~h}$. Caspase- 9 activity was determined and samples were duplicated. The values are representative of three independent experiments. $* P<$ 0.05 . 
results suggest that ROS could facilitate caspase- 9 activation.

\section{$\mathrm{H}_{2} \mathrm{O}_{2}$ induces the activation of caspase-9}

To directly show the role of ROS in caspase-9 activation, HeLa cells were treated with different concentrations of $\mathrm{H}_{2} \mathrm{O}_{2}$, and the auto-cleavage and activity of caspase-9 were subsequently examined. As shown in Figure $2 \mathrm{~A}$, levels of the $35 \mathrm{kDa}$ cleaved fragment and caspase- 9 activity increased in $\mathrm{H}_{2} \mathrm{O}_{2}$-treated cells in a dose-dependent manner. We also determined the level of apoptosis in these cells using Annexin-V/PI flow cytometry and Hoechst 33342/PI double staining, revealing that the $\mathrm{H}_{2} \mathrm{O}_{2}$ treatment did induce apoptosis (Figure 2B).

\section{A}
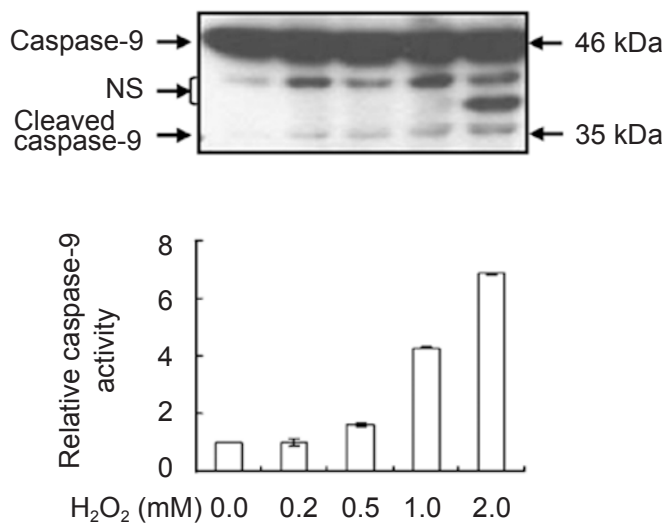

B
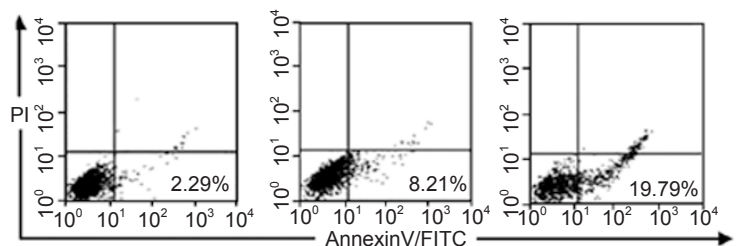

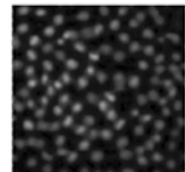

$\mathrm{Oh}$

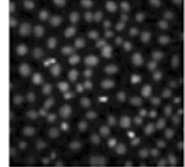

$3 \mathrm{~h}$

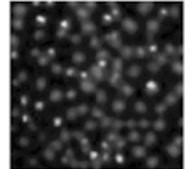

$9 \mathrm{~h}$
Figure $2 \mathrm{H}_{2} \mathrm{O}_{2}$ treatment caused auto-cleavage and activation of caspase- 9 and, consequently, cell apoptosis. (A) HeLa cells were treated with $\mathrm{H}_{2} \mathrm{O}_{2}$ at various concentrations for $5 \mathrm{~h}$. Auto-cleavage fragment ( $35 \mathrm{kDa}$ ) and caspase- 9 activity were determined. NS indicates non-specific bands. Samples were duplicated in each experiment. The values are representative of three independent experiments. (B) HeLa cells were stimulated by $2 \mathrm{mM} \mathrm{H}_{2} \mathrm{O}_{2}$ for various times. Apoptosis rates were monitored by AnnexinV-FITC/PI flow cytometry and Hoechst 33342/PI cell staining. The figures are representative of three experiments.
$\mathrm{H}_{2} \mathrm{O}_{2}$ promotes the disulfide-mediated interaction between caspase-9 and Apaf-1

The caspase- 9 protein contains cysteines that can be targets for oxidative modification by ROS. To determine whether oxidative modification of caspase- 9 occurred in $\mathrm{H}_{2} \mathrm{O}_{2}$-treated cells, F5M staining was performed to examine the oxidative status of caspase-9. As shown in Figure $3 \mathrm{~A}$, the fluorescence of caspase- 9 was reduced significantly in $\mathrm{H}_{2} \mathrm{O}_{2}$-treated cells, indicating that the $\mathrm{H}_{2} \mathrm{O}_{2}$ treatment could induce oxidation of caspase-9.

During the apoptotic process, Apaf-1, caspase- 9 and other components form a complex and together activate caspase-9 activity $[16,18]$. We reasoned that oxidative modifications of caspase- 9 might affect complex formation. The interaction of caspase- 9 and Apaf- 1 was thus examined in $\mathrm{H}_{2} \mathrm{O}_{2}$-treated cells. The oxidative effects of $\mathrm{H}_{2} \mathrm{O}_{2}$ are likely to manifest in the form of disulfide bridges, which may facilitate inter-protein binding. To determine the disulfide-mediated interaction of caspase- 9 and Apaf- 1 in $\mathrm{H}_{2} \mathrm{O}_{2}$-treated cells, co-immunoprecipitation (co-IP) was performed. Cell lysates were immunoprecipitated with an antibody against caspase- 9 and subjected to electrophoresis under either non-reduced or reduced conditions. As shown in Figure $3 \mathrm{~B}, \mathrm{H}_{2} \mathrm{O}_{2}$ treatment promoted the formation of a complex that contained both caspase- 9 and Apaf-1, which could be seen in the non-reduced condition. In the reduced condition, the complex disappeared and the amount of Apaf-1 monomers increased, indicating that the integrity of the complex was maintained by disulfide bridges. A redox diagonal electrophoresis method was also used to detect the formation of intermolecular disulfides (Supplementary information, Figure S1). Immunoblotting for Apaf-1 was performed after the caspase-9 blots were stripped. As shown in Figure 3C, a small fraction of both caspase- 9 and Apaf- 1 appeared at a molecular weight of greater than $175 \mathrm{kDa}$ in the first dimension, whereas their monomeric positions were revealed in the second dimension, at $46 \mathrm{kDa}$ for caspase-9 and at $135 \mathrm{kDa}$ for Apaf-1, indicating that caspase- 9 and Apaf-1 were present in a disulfide-linked complex in $\mathrm{H}_{2} \mathrm{O}_{2}$-treated cells.

Diamide promotes the disulfide-mediated interaction of caspase-9 with Apaf-1 and induces activation of caspase-9 in a mitochondria-free system

ROS can also induce cytochrome $c$ release from mitochondria, which can then bind to Apaf-1 to initiate caspase- 9 activation $[12,19]$. It was thus possible that cytochrome $c$ release induced by $\mathrm{H}_{2} \mathrm{O}_{2}$ might be a mechanism to explain the enhanced interaction of caspase- 9 and Apaf- 1 . To determine the direct effects of oxidative modification on the activation of caspase- 9 , 
A

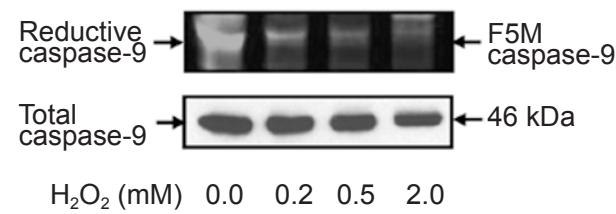

B

IP: Caspase-9

IB: Apaf-1

Caspase-9

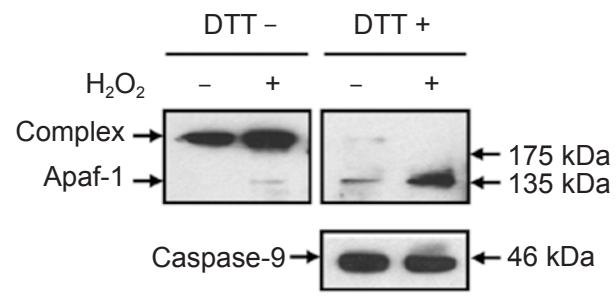

C
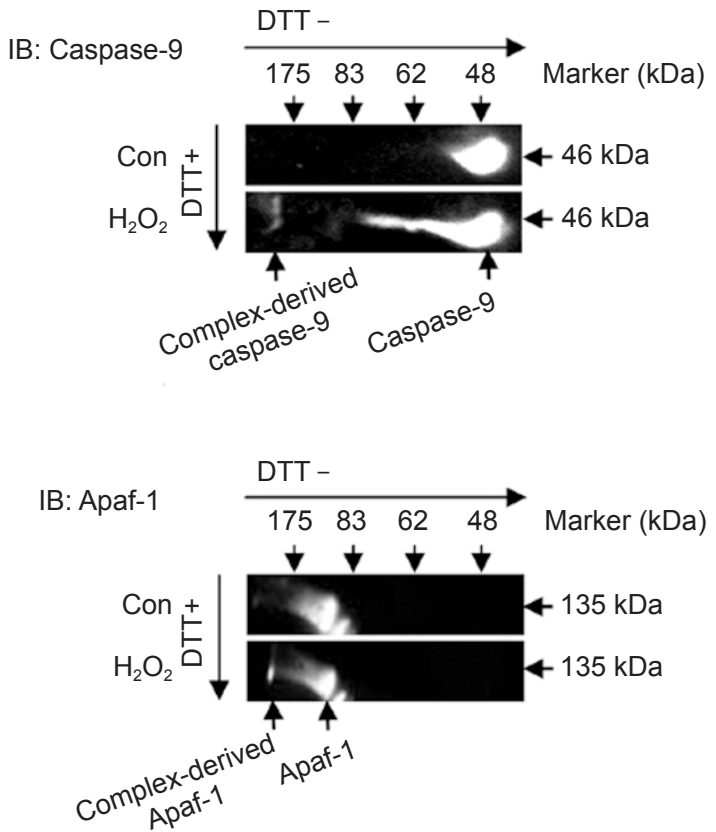

Figure $3 \mathrm{H}_{2} \mathrm{O}_{2}$ induced oxidation of thiols in caspase- 9 and promoted the caspase-9/Apaf-1 interaction by the formation of disulfides in a protein complex. (A) HeLa cells were treated with $\mathrm{H}_{2} \mathrm{O}_{2}$ at various concentrations for $1 \mathrm{~h}$ before incubation with reduced thiol-specific conjugating fluorescence F5M (see Materials and Methods). (B) HeLa cells were exposed to $2 \mathrm{mM} \mathrm{H}_{2} \mathrm{O}_{2}$ for $4 \mathrm{~h}$. The interaction between caspase- 9 and Apaf- 1 was analyzed by co-immunoprecipitation and electrophoresis in non-reducing or reducing conditions before immunoblot. The figures are representative of three experiments. (C) Disulfide-mediated interaction of caspase- 9 and Apaf- 1 in a protein complex was determined by redox diagonal electrophoresis. Immunoblotting for caspase- 9 and Apaf-1 was performed on the same membrane, probing for Apaf- 1 after the caspase- 9 antibody was stripped. The figures are representative of two experiments. we used a mitochondria-free system (Figure 4A). The mitochondria-free cytoplasm was treated with diamide, a thiol oxidant. Caspase- 9 activation as represented by the level of the $35 \mathrm{kDa}$ cleaved fragments could be induced dose-dependently by diamide in these mitochondria-free extracts, which contained equal concentrations of cytochrome $c$ (Figure 4B). The redox diagonal electrophoresis assay (Figure 4C) showed that diamide treatment resulted in caspase-9 (upper) and Apaf-1 (lower) interaction through disulfide bridges. These results suggest that oxidative modifications of caspase- 9 could underlie its ROS-induced activation, which did not depend on increase of cytochrome $c$.

Cysteine 403 is responsible for oxidative modification of caspase-9

Caspase-9 contains 13 cysteine residues [16]. To map the oxidation-sensitive sites responsible for $\mathrm{H}_{2} \mathrm{O}_{2}$-induced activation of caspase-9, we individually mutated 12 of these cysteines to serines, excluding C287, which is required for activation. These caspase- 9 mutants were transfected into cells and followed by $\mathrm{H}_{2} \mathrm{O}_{2}$ treatment. The cell lysates were analyzed by routine and redox diagonal SDS-PAGE electrophoresis followed by IB with an antibody against the His tag. As shown in Figure $5 \mathrm{~A}$, the cleaved fragment of caspase- 9 was markedly decreased in the C403S mutant in comparison with its wild-type counterpart. Consistent with this result, redox diagonal electrophoresis showed no interaction between the $\mathrm{C} 403 \mathrm{~S}$ mutant caspase- 9 and Apaf- 1 in the $\mathrm{H}_{2} \mathrm{O}_{2}$ treated cells (Figure 5B), indicating that $\mathrm{C} 403$ of caspase- 9 was required for the disulfide-based interaction of caspase- 9 with Apaf-1. The other cysteine mutants of caspase-9 displayed similar levels of cleavage and Apaf1 interactions compared with the wild-type protein (data not shown).

To further address the inter-relationship of the disulfide-linked complex with classical apoptosomes, we examined whether the $\mathrm{C} 403 \mathrm{~S}$ mutation affected the interaction of caspase- 9 with cytochrome $c$ or the formation of apoptosomes. After cells were transfected with the wild-type or mutated caspase- 9 constructs and exposed to $\mathrm{H}_{2} \mathrm{O}_{2}$, the exogenous tagged caspase- 9 molecules were pulled down with Ni-IDA columns and IB was performed to detect Apaf-1 and cytochrome $c$ in these extracts. Apoptosome formation was indicated by the presence of Apaf-1 and cytochrome $c$ in the wild-type caspase-9 co-pull-down complex. Significantly less Apaf-1 and cytochrome $c$ were detected in the $\mathrm{C} 403 \mathrm{~S}$ mutant caspase-9 pull-down samples (Figure 5C). These results suggest that oxidative modification of $\mathrm{C} 403$ promotes the disulfide-mediated interaction of caspase-9 with Apaf-1, 
A

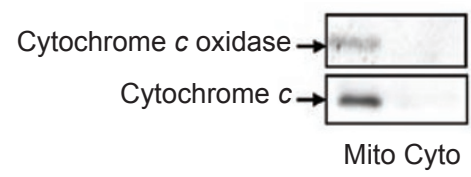

B

Diamide (mM) $\quad 0.0 \quad 0.2 \quad 0.5$

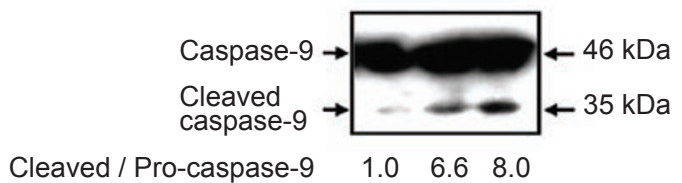

C
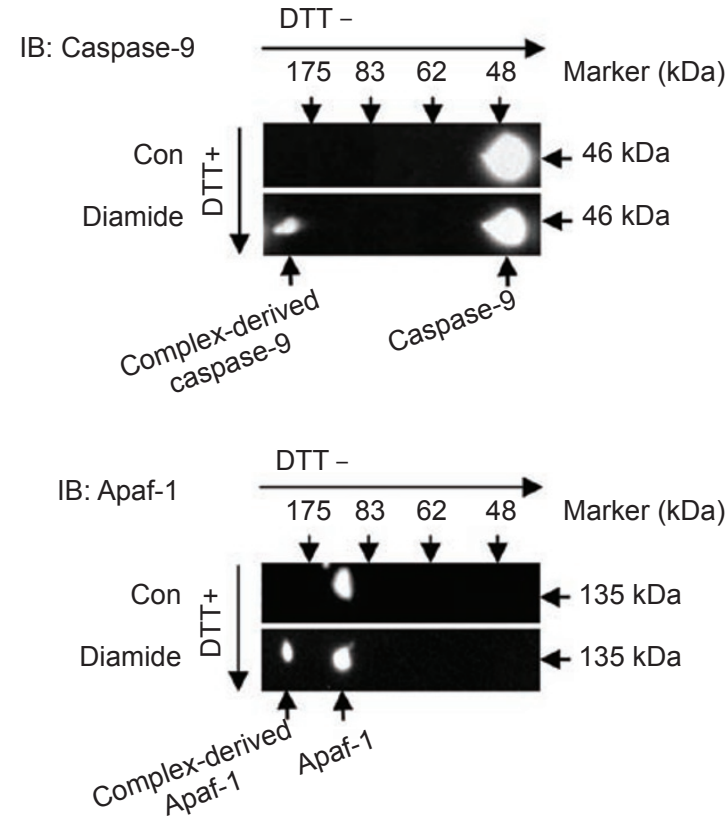

Figure 4 Diamide induced auto-cleavage of caspase-9 and disulfide-mediated interaction of caspase-9/Apaf-1 in an in vitro mitochondria-free system. HeLa cell mitochondria were removed from the cytoplasm to establish mitochondria-free systems. Cytosolic fractions were pre-incubated with thiol-specific oxidant diamide at indicated concentrations at $16^{\circ} \mathrm{C}$ for $30 \mathrm{~min}$, followed by $37^{\circ} \mathrm{C}$ for $4 \mathrm{~h}$ in the presence of $2 \mu \mathrm{g} / \mathrm{ml}$ cytochrome $c$ and $1 \mathrm{mM}$ dATP. (A) Mitochondrial and cytosolic fractions were identified by detection of cytochrome $c$ and cytochrome C oxidase. (B) Auto-cleavage fragments of caspase-9 (35 kDa) were detected and proteins were quantified by densitometry. The extent of caspase- 9 activation is represented as the ratio between cleaved-caspase- 9 and intact caspase-9. (C) Disulfidemediated caspase-9/Apaf-1 interactions were detected with redox diagonal electrophoresis. Immunoblotting for caspase-9 and Apaf-1 was performed on the same membrane, probing for Apaf- 1 after stripping the caspase- 9 antibody. The figures are representative of three experiments. and that this interaction contributes to the formation of the apoptosome that results in caspase- 9 activation.

\section{Discussion}

Several plausible target(s) at which ROS promote apoptosis have been proposed. Voltage-dependent anion channel and cardiolipin can be oxidized [19, 20], which may promote cytochrome $c$ release and collapse of the mitochondrial transmembrane potential. Because ROS may have multiple targets, signaling events that control the balance between pro- and anti-apoptotic factors may also be modulated by oxidative modification of thiols [21,22].

Sato and colleagues have also studied the mechanisms by which ROS promote caspase-9 activation [10]. They showed that ROS positively regulate the apoptotic events downstream of the mitochondrial permeability transition in Jurkat cells; ROS promote apoptosome formation by oxidizing Apaf-1, which in turn helps to activate caspases-9 and -3. They emphasize that oxidation of Apaf-1, not caspase-9, is responsible for the enhancement of apoptosome formation stimulated by ROS. It is notable, however, that caspase-9 is at least not inactivated by ROS in their experiments, because the simple mixture of dATP, cytochrome $c$ and naturally oxidized Apaf-1 and caspase-9 could form the apoptosome.

Despite the fact that ROS can promote or induce caspase- 9 activation by modulating many different factors, as demonstrated by aforementioned studies, the results of the present study indicate that activation of caspase- 9 by ROS largely depends on the disulfide-mediated caspase-9/Apaf-1 interaction. These results imply that oxidative modification of caspase- 9 at the $\mathrm{C} 403$ residue can enhance its interaction with Apaf-1. This effect significantly facilitates apoptosome formation and caspase- 9 activation.

Recent studies indicate the existence of cytochrome $c$-independent mechanism(s) for caspase-9 activation $[23,24]$. Our finding that activity of caspase- 9 can be enhanced by oxidative modification, independently from the increased release of cytochrome $c$, may provide an explanation for caspase-9-related apoptosis in such situations. Nevertheless, the question of whether oxidative modification of caspase- 9 can activate it in the absence of cytochrome $c$ requires further study.

The oxidative sensitivity of specific cysteine residues may impart specificity to ROS-mediated cellular signaling [25], meaning that upon oxidative stress, some proteins will be activated by oxidation, whereas others are inactivated. We postulate that under conditions of oxidative stress, cysteines located at different positions throughout the caspase- 9 protein might show different 
levels of responsiveness. Although the cysteine in the enzymatic active site needs to be maintained in the reduced form for full protease activity, peripheral cysteines, especially those that might be involved in interactions with other proteins, may require oxidization; the activated state would result from the coordinated response.

Sequence analysis shows that there are two cysteines, $\mathrm{C} 12$ and $\mathrm{C} 76$, in the caspase recruitment domain (CARD) of caspase-9 that interact with the CARD of Apaf-1. However, these two cysteines are not involved in the interaction between the two CARD domains [26]. Our

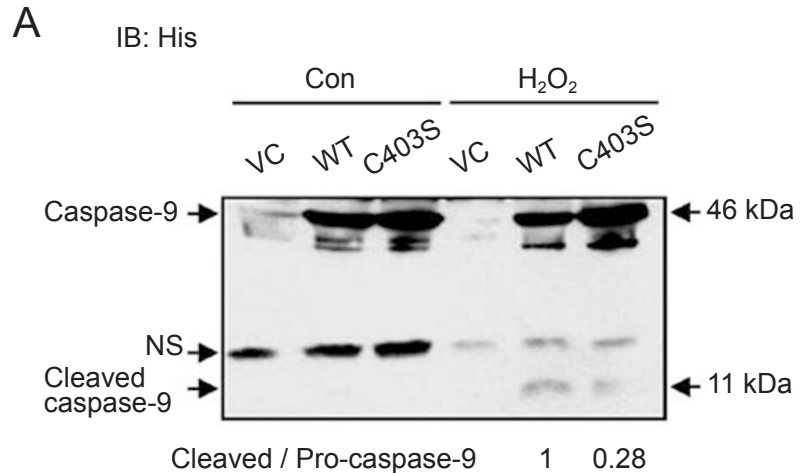

B IB: His
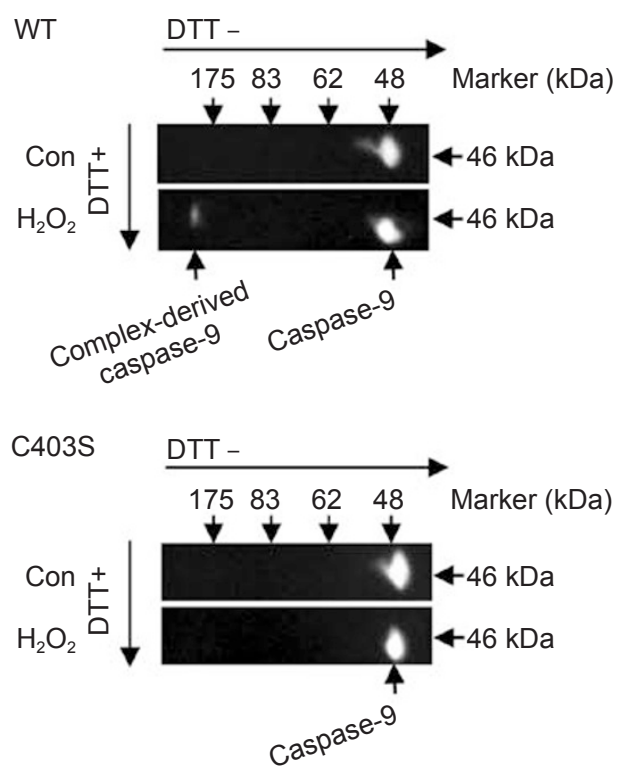

C

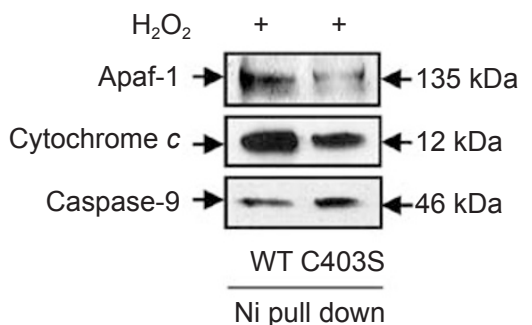

predictions based on structure simulations have excluded the possibility of intramolecular disulfide bond formation between these two cysteines, and indeed, mutations at these sites yield no obvious effects. Out of our expectation, $\mathrm{C} 403$ that does not locate in CARD is responsive to oxidative modification and appears to mediate a disulfide bond upon oxidative modification. Furthermore, the results of redox diagonal electrophoresis indicate that this bond is inter-molecular rather than intramolecular. We therefore postulate that this disulfide bond might bridge C403 of caspase- 9 with a cysteine either in another caspase-9 molecule or in Apaf-1, or perhaps in other proteins that coexist within the same complex, thus promoting the interaction between caspase- 9 and Apaf-1, directly or indirectly. The precise spatial relationship between C403 in caspase-9 and its disulfide partner, as well as the identity of the latter, will be addressed in our future work.

Disulfide bridges have traditionally been thought to be inert covalent linkages between cysteine residues that are formed during protein folding. However, an emerging concept identifies two types of disulfide bonds, a structural group and a redox-sensitive regulatory group, with the latter having direct functional consequences for the cell $[27,28]$. After bacterial transcription factor OxyR was found to be directly activated by thiol oxidation [29], increasing numbers of reports have recently shown that covalent modification of cysteines by disulfide formation can activate proteins in mammalian cells [30-32] and that disulfides are rapidly formed on cell surfaces in response to changes in the cellular redox state [28]. In agreement with these novel concepts, our finding that oxidative modification of caspase- 9 can promote its activation has provided a previously undescribed perspective of how oxidative stress induces apoptosis; in addition to the

Figure 5 Cys 403 mutation blunted the sensitivity of caspase- 9 to $\mathrm{H}_{2} \mathrm{O}_{2}$-induced auto-cleavage and decreased the formation of disulfide-dependent complex and apoptosomes. HeLa cells were transfected with His-caspase-9 (WT) or His-C403S-caspase-9 (C403S). (A) Twenty-four hours after transfection, HeLa cells were treated with $2 \mathrm{mM} \mathrm{H}_{2} \mathrm{O}_{2}$ for $4 \mathrm{~h}$. Auto-cleavage fragments of His-caspase-9 (11 kDa) were detected. The ratio of cleaved-caspase- 9 to intact caspase- 9 is indicated. VC refers to vector control and NS refers to non-specific bands. (B) Twentyfour hours after transfection, HeLa cells were treated with $5 \mathrm{mM}$ $\mathrm{H}_{2} \mathrm{O}_{2}$ for $2 \mathrm{~h}$. The disulfide-dependent complex containing Hiscaspase-9 was detected with redox diagonal electrophoresis and immunoblot. (C) HEK293T cells were transfected with Hiscaspase-9 (WT) or His-C403S-caspase-9 (C403S). Twenty-four hours post transfection, cells were treated with $2 \mathrm{mM} \mathrm{H}_{2} \mathrm{O}_{2}$ for $5 \mathrm{~h}$. Cell lysates were subjected to Ni-IDA pull-down and immunoblot analysis with anti-Apaf-1, anti-cytochrome $c$ and anti-His antibodies. The figures are representative of three experiments. 
ROS-triggered mitochondrial cytochrome $c$ release that activates caspase-9 on the platform of the apoptosome, a direct thiol modification of caspase-9 significantly facilitates its activation in apoptosomes.

\section{Materials and Methods}

\section{Cell culture and reagents}

HeLa and HEK293T cells were maintained in Dulbecco's modified Eagle's medium (DMEM, GibcoBRL, USA) supplemented with antibiotics and $10 \%$ newborn calf serum. Wild-type oral squamous carcinoma cells (OSC-WT) and OSC cells that stably express antisense manganese superoxide dismutase transcripts (OSC-SA) were gifts from Dr T Osaki and T Yamamoto (Kochi University Medical School, Japan) [33]. OSC cells were cultured in 1640 medium (Gibco-BRL). Arsenic trioxide $\left(\mathrm{As}_{2} \mathrm{O}_{3}\right)$ and diamide were purchased from Sigma Chemical Co. (St Louis, MO, USA). TNF- $\alpha$ was purchased from Promega (Madison, USA). Hydrogen peroxide, cytochrome $c$ and dATP were purchased from Sangon (Shanghai, China). F5M, obtained from Molecular Probes (Eugene, USA), was dissolved in dimethylformide to a final concentration of $250 \mathrm{mM}$ and stored at $-20{ }^{\circ} \mathrm{C}$ until use. Caspase- 9 inhibitor LEHD-CHO was purchased from BioVision (Mountain View, USA).

\section{Assay of caspase-9 activity}

The caspase-9 activity detection kit was purchased from Calbiochem (San Diego, USA). Red-LEHD-FMK, a fluorescent marker, binds irreversibly to activated caspase-9 in living cells, such that caspase- 9 activity can be approximated by the fluorescence intensity (excitation $\sim 540 \mathrm{~nm}$; emission $\sim 570 \mathrm{~nm}$ ) detected by flow cytometry. Cells were trypsinized and washed once with complete medium after exposure to different stimuli. Following the addition of $1 \mathrm{ml}$ of Red-LEHD-FMK into $300 \mathrm{ml}$ of cell suspension and incubation for $30 \mathrm{~min}$ to $1 \mathrm{~h}$ in a $37^{\circ} \mathrm{C}$ incubator with $5 \% \mathrm{CO}_{2}$, cells were washed twice and analyzed by flow cytometry (Becton Dickinson, USA).

\section{Annexin-V/propidium iodide (PI) flow cytometry and} Hoechst 33342/PI staining for apoptosis assay

The Annexin-V/PI assay was performed with a flow cytometer (Becton Dickinson) using the FITC-labeled Annexin-V and PI kit (Calbiochem) according to the manufacturer's instructions, as described previously [15]. For cell staining, cells were incubated in complete medium with $5 \mu \mathrm{g} / \mathrm{ml}$ Hoechst 33342 at $37^{\circ} \mathrm{C}$ in the dark for $10 \mathrm{~min}$. PI was co-incubated at $4{ }^{\circ} \mathrm{C}$ in the dark for an additional $10 \mathrm{~min}$. Nuclear condensation was visualized using a fluorescence microscope equipped with a UV-2A filter, and apoptotic cells were identified.

\section{Immunoprecipitation (IP), co-immunoprecipitation (Co-IP) and Ni-IDA pull-down}

IP was performed to extract caspase-9 from cells. Cells were washed once with cold PBS and lysed with cold $1 \times$ IP buffer (1\% Triton X-100, $400 \mathrm{mM} \mathrm{NaCl}, 10 \mathrm{mM}$ Tris, pH 7.4, 1 mM EDTA, $1 \mathrm{mM}$ EGTA ( $\mathrm{pH} 8.0$ ), $0.2 \mathrm{mM}$ sodium ortho-vanadate, $1 \mathrm{mM}$ PMSF, $0.5 \%$ protease inhibitor cocktail, $0.5 \%$ IGEPAL CA-630) at $4{ }^{\circ} \mathrm{C}$ for $30 \mathrm{~min}$, and then lysates were passed several times through a 26-gauge needle and centrifuged at $12000 \times \mathrm{g}$ for 15 $\min$ at $4^{\circ} \mathrm{C}$.

To determine the interaction between caspase- 9 and Apaf-1, co-IP was performed. The samples were carefully prepared so as not to disrupt mitochondria before their removal (as described below for the mitochondria-free system). The supernatants without mitochondria were then used for IP. The supernatants were incubated with the rabbit polyclonal antibody against caspase-9 (1:100, Neomarker, USA) or the rat monoclonal antibody against Apaf-1 (1:200, Chemicon, USA) overnight at $4{ }^{\circ} \mathrm{C}$, followed by incubation with protein A- or protein G-coated agarose beads, respectively (Santa Cruz Biotechnology, USA), for another $4 \mathrm{~h}$ at 4 ${ }^{\circ} \mathrm{C}$. After samples were washed four times with ice-cold PBS and supernatants were removed by centrifugation at $12000 \times g$ for 1 min, casapse- 9 and Apaf- 1 proteins were precipitated individually or co-precipitated if the two molecules were directly or indirectly bound together. The proteins were then separated from the beads using $0.1 \mathrm{M}$ glycine $(\mathrm{pH} 2.5)$ for $15 \mathrm{~min}$ on ice. The supernatants were collected and the $\mathrm{pH}$ was neutralized by the addition of $1 / 10$ volume of $1 \mathrm{M}$ Tris ( $\mathrm{pH} 8.0$ ).

To examine the effect of the caspase-9 mutation, Ni-IDA pull-down was performed after $6 \times$ His-tagged constructs were transfected into HEK293 cells using the His Bind purification kit (Novagen, USA) according to the manufacturer's protocol.

\section{Immunoblot}

Immunoblot was performed for various purposes within these experiments and was accordingly adjusted for different protein samples. To determine the amounts of caspase- 9 and its autocleaved fragments, whole cell lysates and mitochondria-free fractions, respectively, were used. To determine the formation of the caspase-9/Apaf-1 interactive complex in the whole cell system, proteins extracted by co-IP were used. To detect the reactivity of the cysteine-mutant caspase- 9 to apoptotic stimuli, caspase- 9 wildtype or caspase- 9 mutant plasmids with His tags were transfected into HeLa cells and Apaf-1, cytochrome $c$, caspase-9 and its cleavage products were detected. Proteins were loaded onto $10 \%$ or $12 \%$ SDS-PAGE gels, transferred to nitrocellulose membranes, and then detected by the proper primary and secondary antibodies before visualization by enhanced chemiluminescence (Pierce Biotechnology, USA). Various primary antibodies were used, including anti-SOD (1:200, Santa Cruz), anti-procaspase-9 (1:1 000, Chemicon), anti-pancaspase- 9 (targeting the $46 \mathrm{kDa}$ procaspase-9 and the cleaved $35 \mathrm{kDa}$ version, 1:1 000, Neomarker), anti-Apaf-1 (1:1 000, Chemicon), anti-cytochrome $c$ oxidase (1:1 000, Santa Cruz), anti-cytochrome $c$ (1:2 000, Promega), and anti-His (1:1 000, Univ, China).

\section{Assessment of reduced thiols}

To label reduced thiols of protein samples, thiol-specific conjugating fluorescence fluorescein-5-maleimide (F5M) was used according to the previously described method [34]. Caspase-9 within the whole cell system was labeled by F5M by incubating the cells in fresh medium containing $10 \mathrm{mM} \mathrm{F} 5 \mathrm{M}$ for $5 \mathrm{~min}$ at $37^{\circ} \mathrm{C}$ after a $1 \mathrm{~h} \mathrm{H}_{2} \mathrm{O}_{2}$ treatment. Labeled caspase- 9 was retrieved by immunoprecipitation, using the IP buffer containing $1 \mathrm{mM}$ F5M before being subjected to SDS-PAGE. After electrophoresis, the F5M fluorescence of the procaspase- 9 bands on the gel was visualized and photographed using the FLA-5000 Science Imaging System (Fu- 
jifilm, Japan) at an excitation wavelength of $472 \mathrm{~nm}$. Immunoblot was performed using the same gel to determine the total caspase-9.

Sequential two-dimensional non-reducing/reducing SDSPAGE

This method has been referred to as diagonal electrophoresis [35]. Samples were prepared in $1 \times$ SDS sample buffer without any reducing agent (i.e., neither $\beta$-ME nor DTT) and loaded onto $10 \%$ SDS-PAGE gels. After the first dimension, non-reducing electrophoresis, the entire lane containing the separated proteins was excised and incubated in SDS sample buffer containing $100 \mathrm{mM}$ DTT (to reduce any disulfide bonds present between proteins or within proteins) for $20 \mathrm{~min}$. The lane was then rotated $90^{\circ}$ and laid horizontally on top of a large-format 1.5 -mm-thick $12 \%$ acrylamide gel. This electrophoresis in the second reducing dimension would produce a series of dots along a diagonal line. Under these conditions, proteins that were linked by a disulfide bond were separated by DTT treatment and then resolved from one another, migrating further down the gel at their true weight, lower than the diagonal line. Thus, proteins that were linked together via disulfide bonds would be present as a complex at a single position in the first dimension, but would separate and move to their monomeric positions in the second dimension. Finally, immunoblot was performed to display the dots containing Apaf-1 and/or caspase-9 (Supplementary information, Figure S1), and the pictures were shown in reverse as black-to-white.

\section{In vitro mitochondria-free system}

Mitochondria were separated from the cytoplasm by a routine method. Briefly, $5 \times 10^{7}$ cells were collected, washed twice in cold PBS and resuspended in cold RSB $(10 \mathrm{mM} \mathrm{NaCl}, 2.5 \mathrm{mM} \mathrm{MgCl} 2$ and $10 \mathrm{mM}$ Tris- $\mathrm{HCl}, \mathrm{pH} 7.5$ ) containing $1 \mathrm{mM}$ PMSF and $0.5 \%$ protease inhibitor cocktail for 10 min to dilate cells. Samples were then homogenized by 15 passages through a 26 -gauge needle, followed immediately by the addition of $2 / 3$ volume $2.5 \times$ MS buffer $(525 \mathrm{mM}$ mannitol, $175 \mathrm{mM}$ sucrose, $12.5 \mathrm{mM}$ Tris- $\mathrm{HCl}$ and $2.5 \mathrm{mM}$ EDTA, $\mathrm{pH}$ 7.5). Mitochondria were removed by centrifugation of samples at $18000 \times g$ at $4{ }^{\circ} \mathrm{C}$ for $30 \mathrm{~min}$; supernatants represented mitochondria-free fractions. The cytosol and mitochondria were probed by immunoblot for cytochrome $c$ and cytochrome $c$ oxidase; the absence of these two proteins in the cytosolic fraction ensured the purity of the mitochondria-free system.

\section{Site-directed mutagenesis of caspase- 9}

To construct a wild-type caspase- 9 eukaryotic expression vector, caspase- 9 cDNA containing $6 \times$ His tag was amplified by PCR from the pET23b-caspase 9 vector (kindly provided by Drs GS Salvesen and JC Reed, Burnham Institute) using the sense primer 5'-ATG AAT TCT AAG AAG GAG ATA TAC CAT GGA-3' and the antisense primer 5'-TAG GTA CCG TGC TCG CTA GAT TAG TG-3'. The caspase-9 cDNA fragment was subcloned into the EcoRI and $K p n I$ sites of the pFLAG-CMV4 vector. All single or double cysteine mutations of caspase- 9 were generated using the QuikChange site-directed mutagenesis kit (Stratagene, USA) according to the manufacturer's protocol. All mutations were confirmed by DNA sequencing.

\section{Acknowledgments}

We thank Drs T Ozaki and T Yamamoto(Kochi University Medical School, Japan) for their gifts of the wild-type and antisense Mn-SOD OSC cell lines. We also thank Drs GS Salvesen and SJ Riedl (Burnham Institute for Medical Research, USA) for their gift of the caspase-9 construct. Dr Hua Jing (Scripps Research Institute, USA) is especially thanked for her aid with the cysteinepositioning predictions. This work was supported by grants from the National Natural Science Foundation of China (30570965, J Yi; 30600105, J Yang), the Ministry of Sciences and Technologies of China (2006CB910104, J Yi) and the Shanghai Education Committee (05BZ17, J Yang).

\section{References}

1 Georgiou G. How to flip the (redox) switch. Cell 2002; 111:607-610.

2 Lee YJ, Shacter E. Oxidative stress inhibits apoptosis in human lymphoma cells. J Biol Chem 1999; 274:19792-19798.

3 Slee EA, Harte MT, Kluck RM, et al. Ordering the cytochrome c-initiated caspase cascade: hierarchical activation of caspases-2, $-3,-6,-7,-8$, and -10 in a caspase-9-dependent manner. $J$ Cell Biol 1999; 144:281-292.

4 Alnemri ES, Livingston DJ, Nicholson DW, et al. Human ICE/ CED-3 protease nomenclature. Cell 1996; 87:171.

5 Rossig L, Fichtlscherer B, Breitschopf K, et al. Nitric oxide inhibits caspase-3 by S-nitrosation in vivo. J Biol Chem 1999; 274:6823-6826.

6 Mannick JB, Hausladen A, Liu L, et al. Fas-induced caspase denitrosylation. Science 1999; 284:651-654.

7 Torok NJ, Higuchi H, Bronk S, Gores GJ. Nitric oxide inhibits apoptosis downstream of cytochrome $c$ release by nitrosylating caspase 9. Cancer Res 2002; 62:1648-1653.

8 Baker A, Santos BD, Powis G. Redox control of caspase-3 activity by thioredoxin and other reduced proteins. Biochem Biophys Res Commun 2000; 268:78-81.

9 Katoh I, Tomimori Y, Ikawa Y, Kurata S. Dimerization and processing of procaspase-9 by redox stress in mitochondria. $J$ Biol Chem 2004; 279:15515-15523.

10 Sato T, Machida T, Takahashi S, et al. Fas-mediated apoptosome formation is dependent on reactive oxygen species derived from mitochondrial permeability transition in Jurkat cells. J Immunol 2004; 173:285-296.

11 Moungjaroen J, Nimmannit U, Callery PS, et al. Reactive oxygen species mediate caspase activation and apoptosis induced by lipoic acid in human lung epithelial cancer cells through Bcl-2 down-regulation. J Pharmacol Exp Ther 2006; 319:1062-1069.

12 Andoh T, Chock PB, Chiueh CC. The roles of thioredoxin in protection against oxidative stress-induced apoptosis in SHSY5Y cells. J Biol Chem 2002; 277:9655-9660.

13 England K, Cotter TG. Direct oxidative modifications of signalling proteins in mammalian cells and their effects on apoptosis. Redox Rep 2005; 10:237-245.

14 Yi J, Yang J, He R, et al. Emodin enhances arsenic trioxideinduced apoptosis via generation of reactive oxygen species and inhibition of survival signaling. Cancer Res 2004; 64:108116. 
15 Yang J, Li H, Chen YY, et al. Anthraquinones sensitize tumor cells to arsenic cytotoxicity in vitro and in vivo via reactive oxygen species-mediated dual regulation of apoptosis. Free Radic Biol Med 2004; 37:2027-2041.

16 Li P, Nijhawan D, Budihardjo I, et al. Cytochrome $c$ and dATP-dependent formation of Apaf-1/caspase-9 complex initiates an apoptotic protease cascade. Cell 1997; 91:479-489.

17 Riedl SJ, Salvesen GS. The apoptosome: signalling platform of cell death. Nat Rev 2007; 8:405-413.

18 Shi Y. Caspase activation: revisiting the induced proximity model. Cell 2004; 117:855-858.

19 Madesh M, Hajnoczky G. VDAC-dependent permeabilization of the outer mitochondrial membrane by superoxide induces rapid and massive cytochrome $c$ release. J Cell Biol 2001; 155:1003-1015.

20 Kagan VE, Tyurina YY, Bayir H, et al. The "pro-apoptotic genies" get out of mitochondria: oxidative lipidomics and redox activity of cytochrome c/cardiolipin complexes. Chem Biol Interact 2006; 163:15-28.

21 Biswas S, Chida AS, Rahman I. Redox modifications of protein-thiols: emerging roles in cell signaling. Biochem Pharmacol 2006; 71:551-564.

22 Kamata $\mathrm{H}$, Honda $\mathrm{S}$, Maeda $\mathrm{S}$, et al. Reactive oxygen species promote TNFalpha-induced death and sustained JNK activation by inhibiting MAP kinase phosphatases. Cell 2005; 120: 649-661.

23 Hao Z, Duncan GS, Chang CC, et al. Specific ablation of the apoptotic functions of cytochrome $c$ reveals a differential requirement for cytochrome $c$ and Apaf-1 in apoptosis. Cell 2005; 121:579-591.

24 Yu X, Wang L, Acehan D, Wang X, Akey CW. Three-dimensional structure of a double apoptosome formed by the Drosophila Apaf-1 related killer. J Mol Biol 2006; 355:577-589.

25 Barford D. The role of cysteine residues as redox-sensitive regulatory switches. Curr Opin Struct Biol 2004; 14:679-686.

26 Nicholson DW. Caspase structure, proteolytic substrates, and function during apoptotic cell death. Cell Death Differ 1999; 6:1028-1042.

27 Hogg PJ. Disulfide bonds as switches for protein function. Trends Biochem Sci 2003; 28:210-214.

28 Yang Y, Song Y, Loscalzo J. Regulation of the protein disulfide proteome by mitochondria in mammalian cells. Proc Natl Acad Sci USA 2007; 104:10813-10817.

29 Zheng M, Aslund F, Storz G. Activation of the OxyR transcription factor by reversible disulfide bond formation. Science 1998; 279:1718-1721.

30 Lin D, Takemoto DJ. Oxidative activation of protein kinase Cgamma through the $\mathrm{C} 1$ domain. Effects on gap junctions. $J$ Biol Chem 2005; 280:13682-13693.

31 Macpherson LJ, Dubin AE, Evans MJ, et al. Noxious compounds activate TRPA1 ion channels through covalent modification of cysteines. Nature 2007; 445:541-545.

32 Burgoyne JR, Madhani M, Cuello F, et al. Cysteine redox sensor in PKGIa enables oxidant-induced activation. Science 2007; 317:1393-1397.

33 Ueta E, Yoneda K, Yamamoto T, Osaki T. Manganese superoxide dismutase negatively regulates the induction of apoptosis by 5 -fluorouracil, peplomycin and gamma-rays in squamous cell carcinoma cells. Jpn J Cancer Res 1999; 90:555-564.

34 Jing Y, Yang J, Wang Y, et al. Alteration of subcellular redox equilibrium and the consequent oxidative modification of nuclear factor kappaB are critical for anticancer cytotoxicity by emodin, a reactive oxygen species-producing agent. Free Radic Biol Med 2006; 40:2183-2197.

35 Sommer A, Traut RR. Diagonal polyacrylamide-dodecyl sulfate gel electrophoresis for the identification of ribosomal proteins crosslinked with methyl-4-mercaptobutyrimidate. Proc Natl Acad Sci USA 1974; 71: 3946-3950.

(Supplementary information is linked to the online version of the paper on the Cell Research website.) 